$15^{\text {th }}$ International Conference on

AEROSPACE SCIENCES \& AVIATION TECHNOLOGY,

$\boldsymbol{A S A T}$ - 15 - May 28 - 30, 2013, Email: asat@ mtc.edu.eg, Military Technical College, Kobry Elkobbah, Cairo, Egypt, Tel: +(202) 24025292 -24036138, Fax: +(202) 22621908

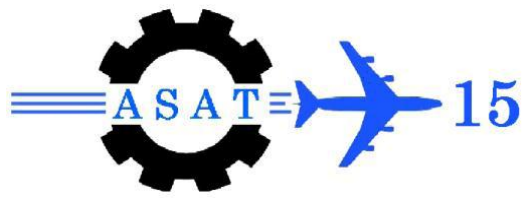

\title{
Thermal Image Assessment using CFD Analysis
}

\author{
\{A. M. Saleh, A. S. El-Wetedy $\}^{*}$
}

\begin{abstract}
Thermal image is the figure presenting the captured thermal energy radiation of the distinct points of the outer surface of anybody due to their temperature. The thermal status of few elemental-geometrical shaped bodies has been described. The physical models of these cases were established. CFD technique is applied to compute the temperature distribution field on the outer surfaces of the body. The same data package FLUENT was used to map related images to these temperature distributions. Then the thermal images of the same cases under the prescribed seen conditions have been captured using thermal camera. Comparison analyses have been carried out. It was concluded that the technique itself is valid. However, too much attention should be paid to the coincidence of constructive factors, in addition to the run variable parameters such as the spectral emissivity.
\end{abstract}

Keywords: Thermal radiation, thermal image, infrared camera.

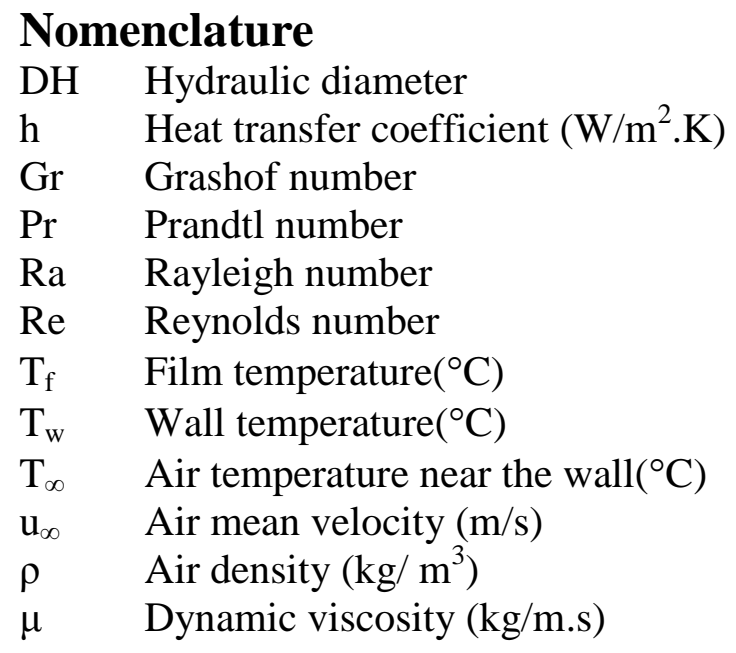

\section{Introduction}

Thermal images captured by infrared cameras are extensively used in enormous applications. The detection of objects using thermal images presents great importance in industry like heat treatment of metal alloys, engine tests and performance, inspection of generators, cutting, rolling, etc. Other applications are also in agriculture and food industry, in addition to environmental, medical, and scientifically research [1].

Field arrangements and setups are expensive and provide limited information against the numerous possible scenarios in which the system should operate. And as relatively cheaper cost, simulation provides more comprehensive statistical pictures. Although confidence might 
be relatively diminished in the absence of the real test. The growing power of computers and the increasing expense of field data collections, the simulation of signatures is becoming more important and more feasible [2].

There are different commercial packages in this field, like those of thermo-analytics company (Rad therm, Win therm, Muses, etc.). Efforts should concentrate on expanding the knowledge in the simulation modes to create a thermal image. The engagement of these models give the quantities forming the thermal image in the effect of solar radiation, sky radiance, background radiance, environmental effects, and heat exchange among the whole site components. Concentrate on heat transfer model and its sub-model (geometry model, thermal model, surrounding model) give quantities forming thermal image at zero range captured by a $100 \%$ effective sensor.

The physical model of a general object has been described through the establishment of the set of three comprehensive conditions (geometrical, thermal, and fluid dynamics). The effect of geometry, shapes, and configurations together with the effect of material properties and surrounding flow conditions are liked and solved by the package (FLUENT 6.3 code) forming the computed temperature distribution on the objects surfaces in a figure of thermal image for objects. This numerical formulation for the heat transfer problem presents the theoretical study in this work, which should be supported with an experimental work by using thermal camera to set up image. Selected objects were studied (cube and prism), then analyzing the results of the comparison between produced images for every matched case in the theoretical and experimental work. These analyses will clear up the causes of errors between the two matched images in every case, and also will clear up the effects of some parameters in producing the thermal image.

The work here will focus on the effect of two main changes (shape and material), other conditions have been fixed. In addition basic geometrical shapes only were chosen for presentation.

\section{Computational Models}

This study will be proceeded to achieve its objective through studying three models case involving the changing of object shapes and materials with fixing for other conditions. The shapes are cube, and prism. The materials are steel and brass. The reasons for selecting these two kinds of shapes depend on that these shapes are basic in geometrical configuration for most objects. The reasons for the selection these two kinds of materials are that they are from the most common materials in our life. The objects is configured in dimensions such that to avoid complications in computations. The models are in three dimensions to study the effect of heat exchange between the faces of objects and the surroundings. The temperature on the model surfaces ranged from $30^{\circ} \mathrm{C}$ to $90^{\circ} \mathrm{C}$. This range of temperature will agree with temperature range of most common terrestrial objects $(8 \mu \mathrm{m}-12 \mu \mathrm{m})$. The flow conditions are defined as a flow of air in ambient temperature, and velocity of air surrounding the object to show the effect of turbulence which related to the Reynolds number $(\mathrm{Re})$. The models are computed in the package of FLUENT 6.3 to get the temperature distribution on objects surfaces. This set-up involves physical, mathematical, and numerical formulation for the models; these formulations are presented as follows. 


\subsection{Physical Formulation}

The first model is configured geometrically where the object's shape is hollow cube with length 0.3 meter, and made from steel. The heat source's shape is cylinder with diameter 0.08 meter, and length 0.09 meter and is hanged up in the cube canter. The source produces quantity of heat equal to 100 Watt. The object is surrounded with flow of air at a mean velocity of $2.2 \mathrm{~m} / \mathrm{s}$ and ambient temperature. The model with its surrounding conditions is enclosed in a parallelogram enclosure which opened from front surface. The enclosure has dimensions of $2 \mathrm{~m} \times 1 \mathrm{mx} 1 \mathrm{~m}$. Table (1), figures (1), and (2) describe the three models and the physical formulation.

\section{Table (1): Models Descriptions}

\begin{tabular}{l|l|l}
\hline \hline 1 & Cube-steel-base & $\begin{array}{l}\text { Shape: Cube, Material: Steel, Heat source position: near the base } \\
\text { center of object }\end{array}$ \\
\hline 2 & Prism-steel-base & $\begin{array}{l}\text { Shape: Prism, Material: Steel, Heat source position: near the } \\
\text { base center of object }\end{array}$ \\
\hline 3 & Cube-brass-base & $\begin{array}{l}\text { Shape: Cube, Material: Brass, Heat source position: near the } \\
\text { base center of object }\end{array}$ \\
\hline \hline
\end{tabular}

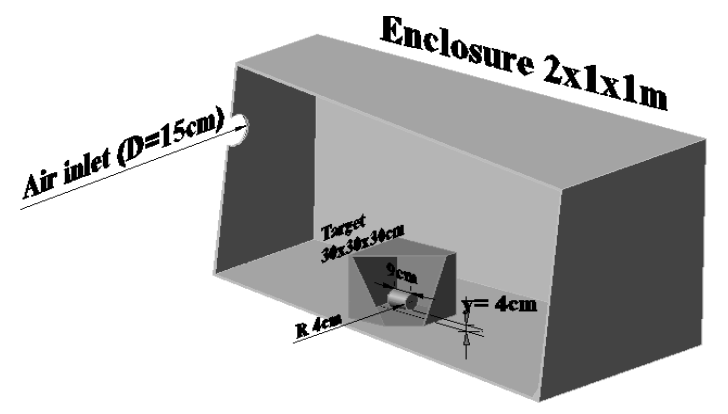

Figure (1): Physical formulation of $2^{\text {nd }}$ model

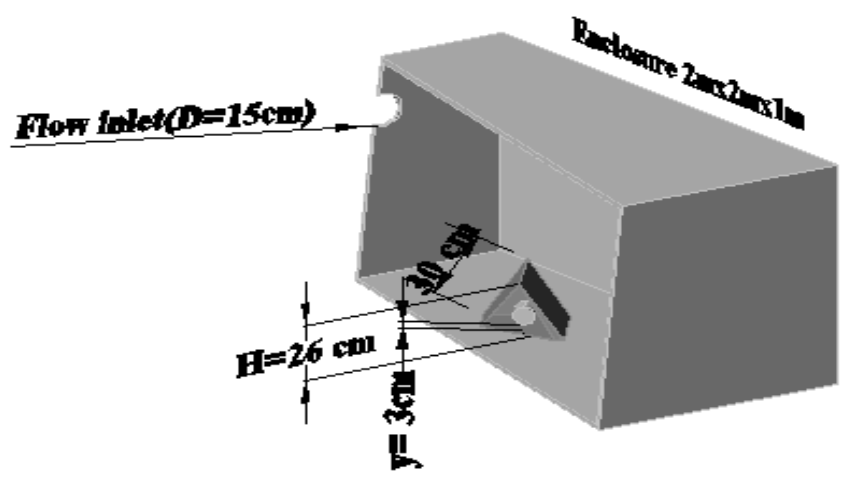

Figure(2): Physical formulation of $1^{\text {st }}, 3^{\text {rd }}$ models

\subsection{Computational Formulation}

The computational formulations use the package of (FLUENT 6.3 code). This code is based on a finite volume technique and uses a set of equations in the finite difference form. In this study the governing equations that solve the problem are the continuity, the momentum, the energy, and radioactive equations. Some dimensional and dimensionless values are evaluated. These values are the properties of air inside the target and enclosure, the Reynolds number 
(Re) to define if the flow is in the turbulence or laminar, the Grashof number (Gr) to define if the heat transfer by convection is free or forced convection, and also the heat transfer coefficient $(\mathrm{h})$ will be estimated. These values are differ from one case to the other and are presented as follows:

\section{a. Cube-steel-base}

\section{Estimating air properties in enclosure and at walls:}

The film temperature at enclosure walls is:

$T_{f}=\frac{T_{\infty}+T_{w}}{2}=35^{\circ} C=308^{\circ} \mathrm{K}$

Assume the problem of flow in enclosure is a flow in a duct, so $\boldsymbol{D}_{\boldsymbol{H}}=1 \boldsymbol{m}$, and assume the mean velocity of flow $\boldsymbol{u}_{\infty}=2.2 \mathrm{~m} / \mathrm{s}$

$$
\left.\boldsymbol{R}_{e}=\frac{\rho \boldsymbol{u}_{\infty} \boldsymbol{D}_{H}}{\boldsymbol{\mu}}=\frac{(1.149)(2.2)(1)}{\left(1.998 \times 10^{-5}\right)}=126516.5\right\rangle 4000 \quad \text { (Turbulent flow) }
$$

h: Heat transfer coefficient will be estimated as heat transferred by free convection at the model walls and its value is about. $\boldsymbol{h}=6 \boldsymbol{W} / \boldsymbol{m}^{2} \cdot \boldsymbol{K}$

The flow inlet mean velocity is $\boldsymbol{u}_{\infty}=5.5 \mathrm{~m} / \mathrm{s}$

$\operatorname{Re}=47443.7$

\section{Estimating air properties inside the object:}

$\boldsymbol{R} \boldsymbol{a}=\boldsymbol{G}_{\boldsymbol{r}} . \boldsymbol{P}_{\boldsymbol{r}}=\frac{(9.8) \cdot(0.0028986) \cdot(6) \cdot(0.6)^{3} \cdot(0.7032)}{\left(2.076 \times 10^{-5}\right)^{2}}=0.6 \times 10^{8} \quad$ (Laminar flow)

Rayleigh numbers less than 108 indicate a buoyancy-induced laminar flow, with the transition to turbulence which occurs over the range of $108<\mathrm{Ra}<1010$. [3], [4].

\section{b. Prism-steel-base}

Estimating air properties in enclosure and at walls:

$$
\boldsymbol{T}_{f}=308^{\circ} \mathrm{K}
$$

Assume the problem of flow in enclosure is a flow in a duct, so $\boldsymbol{D}_{\boldsymbol{H}}=1 \boldsymbol{m}$, and assume the mean velocity of flow $\boldsymbol{u}_{\infty}=2.2 \mathrm{~m} / \mathrm{s}$

$\boldsymbol{R}_{\boldsymbol{e}}=126516.5>4000$ (Turbulent flow)

$h$ : Heat transfer coefficient will be estimated as heat transferred by free convection at the model walls and its value is about $\boldsymbol{h}=6 \boldsymbol{W} / \boldsymbol{m}^{2} . K$.

The flow inlet mean velocity is $\boldsymbol{u}_{\infty}=5.5 \mathrm{~m} / \mathrm{s}$

$\boldsymbol{R e}=47443.7$

\section{Estimating air properties inside the object:}

$\boldsymbol{R} \boldsymbol{a}=0.75899 \times 10^{8}$ (Laminar flow)

Rayleigh numbers less than $10^{8}$ indicate a buoyancy-induced laminar flow, with the transition to turbulence which occurs over the range of $10^{8}<\mathrm{Ra}<10^{10}[\underline{3}]$, [4]. 


\section{c. Cube-brass-base}

\section{Estimating air properties in enclosure and at walls:}

$\boldsymbol{T}_{f}=306^{\circ} \mathrm{K}$

Assume the problem of flow in enclosure is a flow in a duct, so $\boldsymbol{D}_{\boldsymbol{H}}=1 \boldsymbol{m}$, and assume the mean velocity of flow $\boldsymbol{u}_{\infty}=2.2 \mathrm{~m} / \mathrm{s}$

$$
\left.\boldsymbol{R}_{\boldsymbol{e}}=212021.01\right\rangle 4000 \quad \text { (Turbulent flow) }
$$

$h$ : Heat transfer coefficient will be estimated as heat transferred by free convection at walls and its value is about $\boldsymbol{h}=6 \boldsymbol{W} / \boldsymbol{m}^{2} \cdot \boldsymbol{K}$.

The flow inlet mean velocity is $\boldsymbol{u}_{\infty}=5.5 \mathrm{~m} / \mathrm{s}$

$\boldsymbol{R e}=47824.35$

\section{Estimating air properties inside the object:}

$\boldsymbol{R} \boldsymbol{a}=0.769 \times 10^{8}$ (Laminar flow)

Rayleigh numbers less than $10^{8}$ indicate a buoyancy-induced laminar flow, with the transition to turbulence which occurs over the range of $10^{8}<\mathrm{Ra}<10^{10}$ [3], [4].

\section{Computational Procedure}

The package FLUENT 6.3 solves any sets of equations problems numerically in the form of finite difference equations used in famous discretization schemes of CFD techniques. For solving the models in this package, the computational work is as follows:

i. Setup the models with the previous physical conditions in CAD system tool associated with the package and called GAMBIT as follows :

- The drawings are in 3D dimensions and the units in meters.

- The optimum mesh size of the cells differs in volumes assisted for the models to make the optimum convergence and reduce the errors of the solution.

- The optimum mesh size for objects and heat source cells are about $0.02 \mathrm{~m}$.

- The optimum mesh size of enclosure cells is $0.1 \mathrm{~m}$.

And to ensure the selection of the mesh size, a convergence study has been done for this purpose. This convergence study was done on one case by comparing the results of temperatures between two generated mesh size $(0.02 \mathrm{~m}$, and $0.04 \mathrm{~m})$. An experimental work was prepared to judge the accurate results for the two selected mesh size, figure (3a), and figure (3b) show the results for this Convergence study.

ii. Specify various parameters associated with the solution method which used in the calculation:

- Specify the numerical method (pressure-based solver), which uses a segregated algorithm to solve the individual governing equations for the solution variables (e.g. $u$, $v, w, p, T, k, \varepsilon$ etc.) one after another. This algorithm is memory-efficient; however, the solution convergence is relatively slow. Also using the coupled algorithm, by coupling momentum and continuity equations which are solved in a closely coupled manner in the same pressure-based solver. The rate of solution convergence significantly improves when compared to the segregated algorithm; however the memory requirement increases by $1.5-2$ times that of the segregated algorithm. 


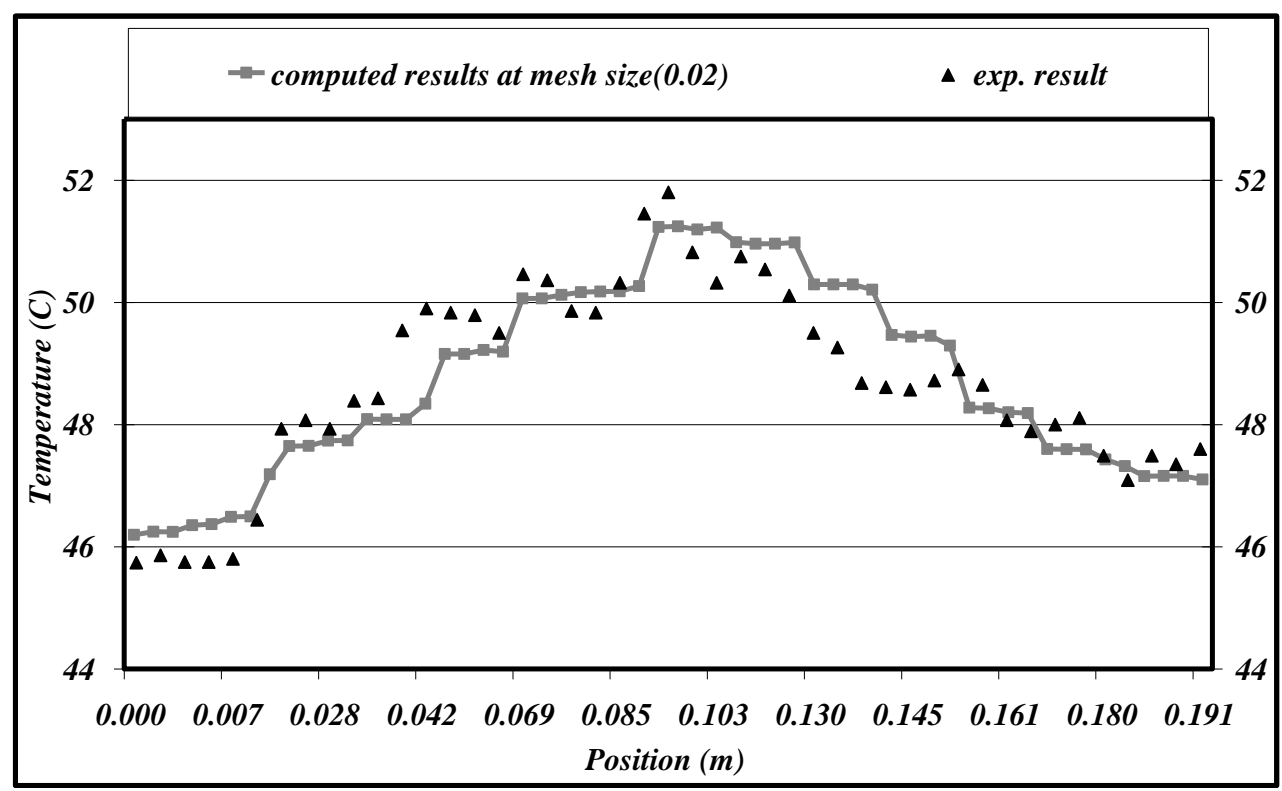

Figure (3a): The convergence study on mesh size of $0.02 \mathrm{~m}$

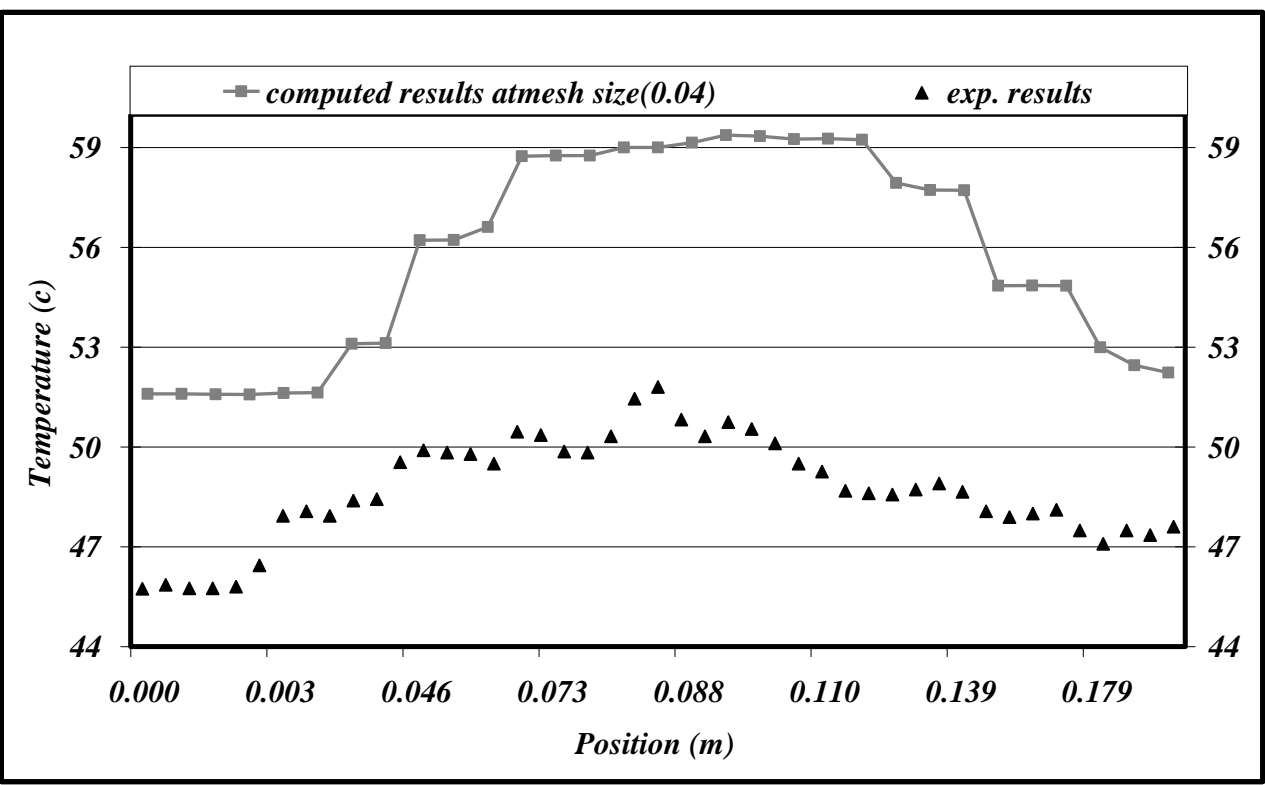

Figure (3b): The convergence study on mesh size of $0.04 m$

- Specify the domain space (3D), specify the time independency (steady-state flow), specify the velocity formulation (Absolute-velocity formulation) used because the flow in the domain is not rotating, and specify the gradient option (Green-gauss nodebased), the node-based averaging scheme is known to be more accurate for unstructured meshes, most notably for triangular and tetrahedral meshes. 
- Define the turbulent flow by using the RNG k- $\varepsilon$ model (found a wide acceptance and seems particularly applicable to problems involving moderate to high Reynolds numbers), and enter the parameters of turbulent flow

- Specify the used radiation models (DTRM: Discrete transfer radiation model).The main assumption of the DTRM is that the radiation leaving the surface element in a certain range of solid angles can be approximated by a single ray. The ray tracing technique used in the DTRM can provide a prediction of radiative heat transfer between surfaces without explicit view-factor calculations. The accuracy of the model is limited mainly by the number of rays traced and the computational grid. This model has some advantages which make it more suitable for solving the models; DTRM is computationally very expensive when there are too many surfaces to trace rays from and too many volumes crossed by the rays. To reduce the computational time, the number of radiating surfaces and absorbing cells is reduced by clustering surfaces and cells into surface and volume "clusters".

- Specify the boundary conditions. Temperatures, heat fluxes, and flow inlet velocity all can be used for boundary conditions. To run three-dimensional simulation requires boundary conditions for the five enclosure surfaces (left, right, top, bottom, back boundaries) which are need temperatures values, heat transfer coefficients, emissivity of solid material of enclosure faces and boundary conditions for the flow inlet opening in the left face of enclosure and flow outlet at the front of the enclosure, which needs flow velocity, turbulence parameters, flow temperature, and flow emissivity, all these boundaries will be defined for each model alone. For the object's boundary conditions which owns coupled faces, this coupling means that the solid zone of the object's faces have a fluid zone on each side, which helps in contributing the effects of the fluid zones on object's faces, these boundaries need only thermal conditions (material emissivity). For the heat source boundary condition, it needs heat flux generated from it. For initial condition, the same initial condition for all models will be the system starts (flow inlet); this initial condition helps in reaching the convergence quickly.

iii. Setup the solution parameters:

- Setup the under-relaxation factors; the values of these factors are reduced for the turbulent flows or high-Rayleigh-number natural-convection problems. Using firstorder momentum discretization and for much skewed meshes the decreasing of the explicit relaxation to 0.5 is enough. Setting the courant number (CFL) to 20 is enough for convergence.

- Setup the Discretization schemes involves using the PRESTO! Scheme (Pressure discretization scheme) for flows with high-Rayleigh-number natural convection. Using for other discretization schemes the first-order upwind scheme is enough for the best convergence of the solution and accurate result. 


\section{Discussion of CFD Results}

Computational solution gives the following results as shown in figures $(4,5$, and 6$)$.. The configuration of the models is illustrated in figures (1) and (2). Figure (4) shows the result of the first model. The object of interest is a cubic shape and its material is steel. The heat source position is in the center of the object near the base of the object with quantity of heat about $100 \mathrm{~W}$ at boundary condition. The velocity of flow at the inlet of enclosure opening is 5.5 $\mathrm{m} / \mathrm{s}$. The results show the filled contours of temperature on the objects surfaces and the enclosure walls. The range of temperature is $\left(32^{\circ} \mathrm{C} \sim 67^{\circ} \mathrm{C}\right)$. Figure (5) shows the result of the second model. The object of interest is a prism shape and its material is steel. The heat source position is in the center of the object near the base of the object with quantity of heat about $100 \mathrm{~W}$ at boundary condition. The velocity of flow at the inlet of enclosure opening is 5.5 $\mathrm{m} / \mathrm{s}$. The results show the filled contours of temperature on the objects surfaces and the enclosure walls. The range of temperature is $\left(32^{\circ} \mathrm{C} \sim 90^{\circ} \mathrm{C}\right)$. Figure (6) shows the result of the third model. The object of interest is a cubic shape and its material is brass. The heat source position is in the center of the object near the base of the object with quantity of heat about $100 \mathrm{~W}$ at boundary condition. The velocity of flow at the inlet of enclosure opening is 5.5 $\mathrm{m} / \mathrm{s}$. The result shows the filled contours of temperature on the objects surfaces and the enclosure walls. The range of temperature is $\left(32^{\circ} \mathrm{C} \sim 60^{\circ} \mathrm{C}\right)$. To get confidence in computational results, an experimental study has been carried out on real configurations similar to the setup of these modes. Analysis and comparisons have been carried out between the computation and experimental work. These comparisons has been done by studying the temperature distribution on matched isothermal lines in both two images to show the errors in the lines trends and values of temperatures. The experimental work is submitted and explained in the following section.

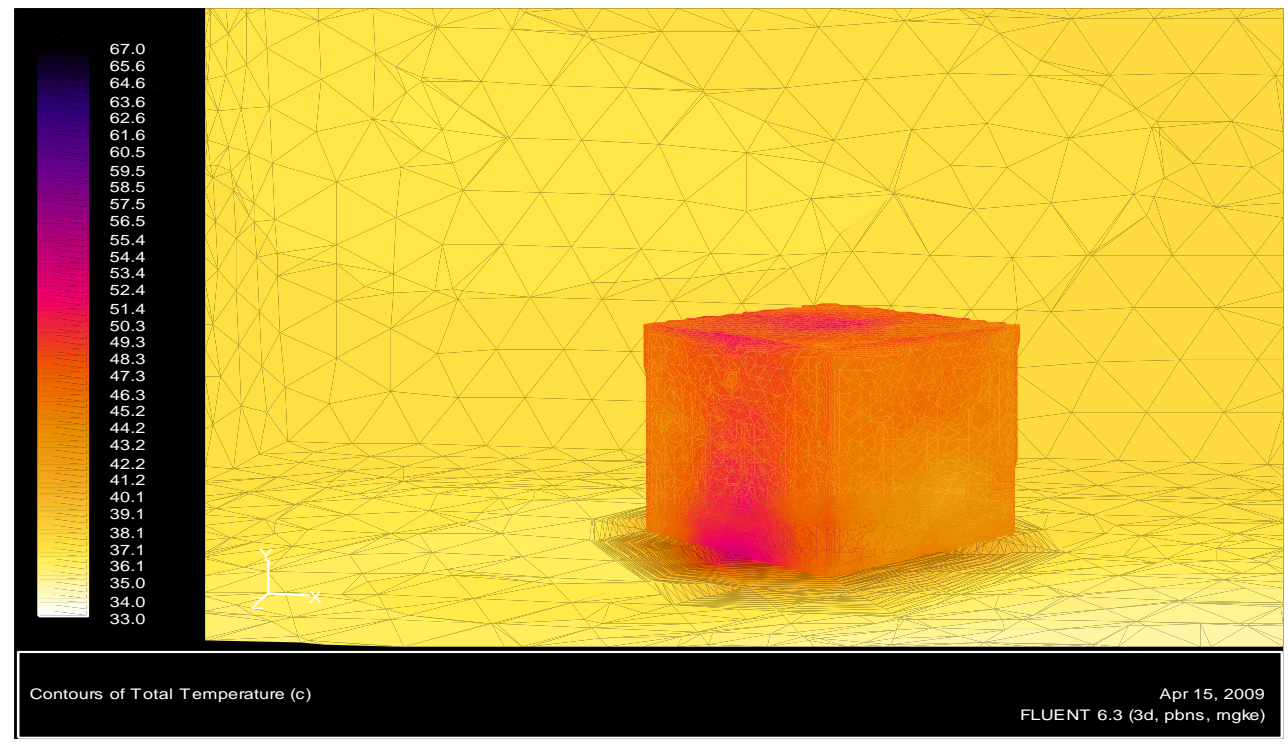

Figure (4): The colored picture of temperature distribution of 1st computed model. 


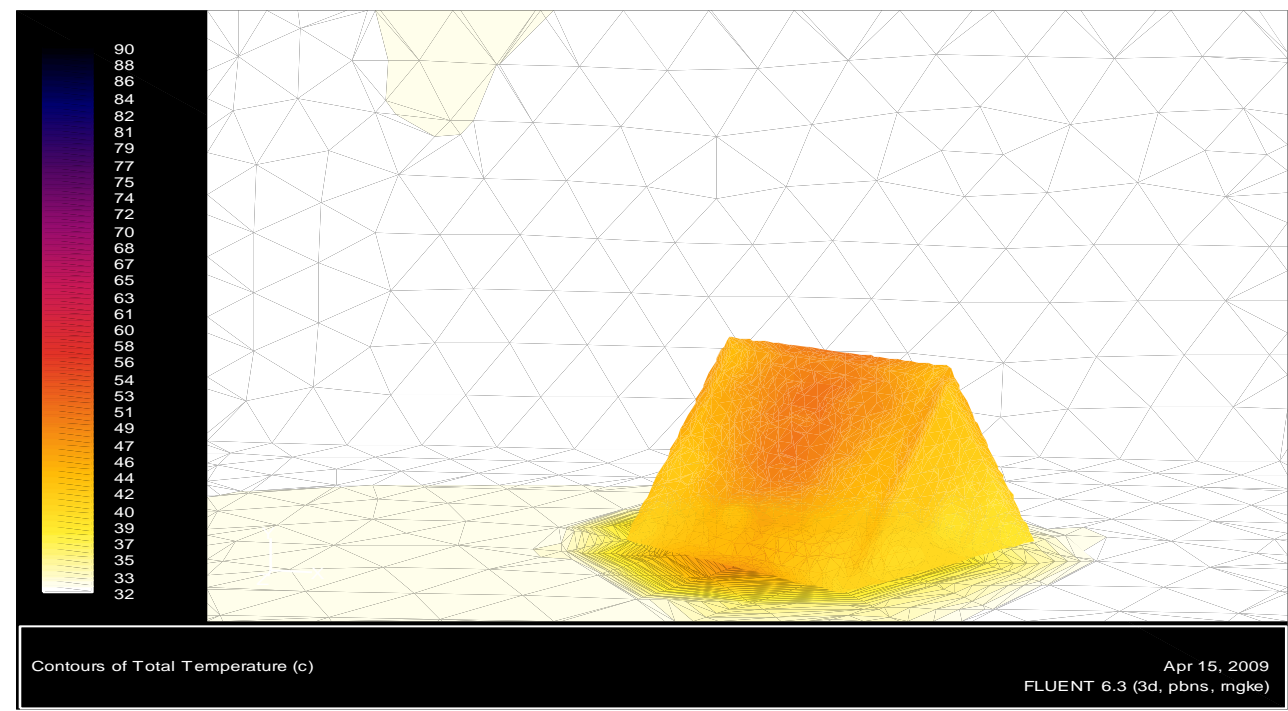

Figure (5): The colored picture of temperature distribution of 2 nd computed model

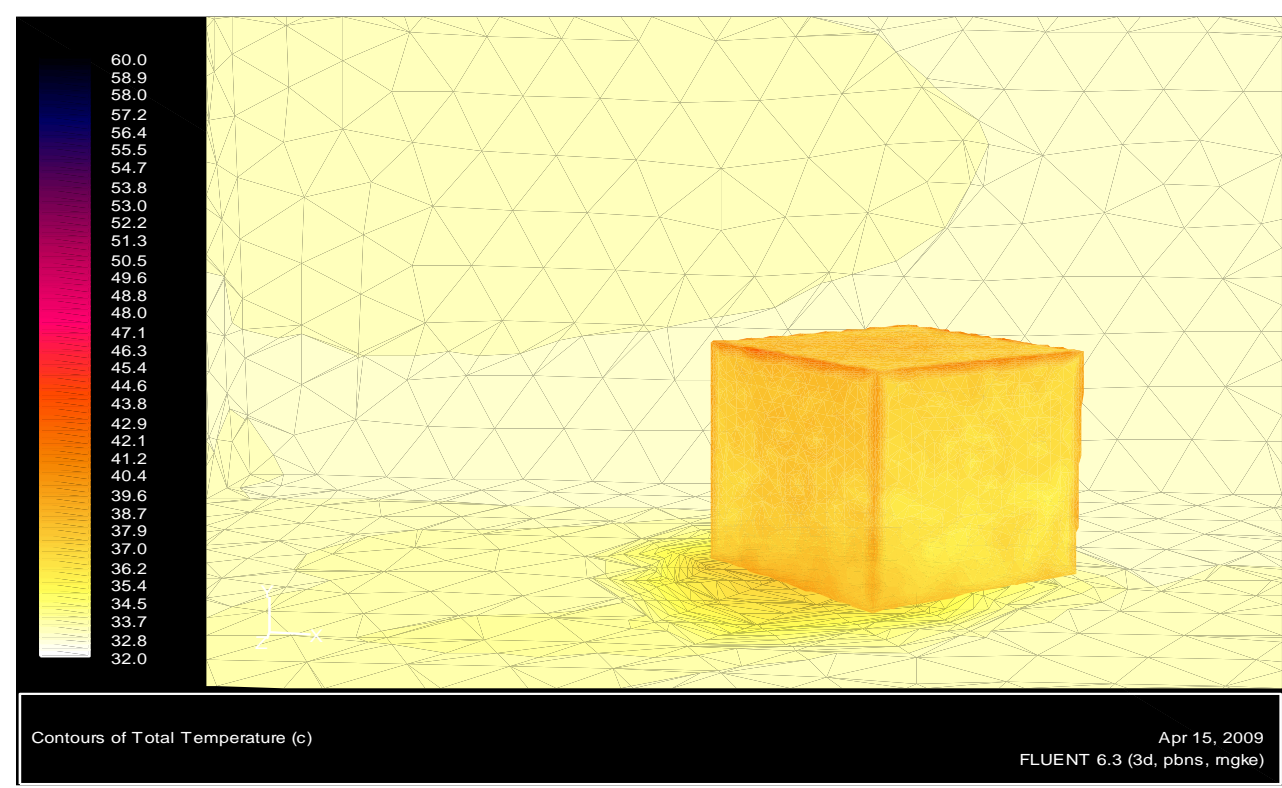

Figure (6): The colored picture of temperature distribution of 3rd computed model.

\subsection{Experimental Work and Analysis}

The experimental setup involves the preparation the models and enclosure with the same dimensions and conditions used in mathematical models. Figures (7) and (8) show a schematic drawing and photo for the experimental setup. The system includes:

1- Voltage regulator (VARIAC).

2- Wattmeter.

3- Digital thermocouple reader.

3- IR thermometer.

4- Thermal camera ( Model SC2000, FLIR systems).

5- Air blower and speedometer. 
The thermal camera is used to get the thermal image for the different models at the specified conditions for each case which adapted with the mathematical model. In addition to the thermal image produced from the thermal camera, the digital thermometer measures the temperatures at selected points to verify the captured thermal image by the camera.

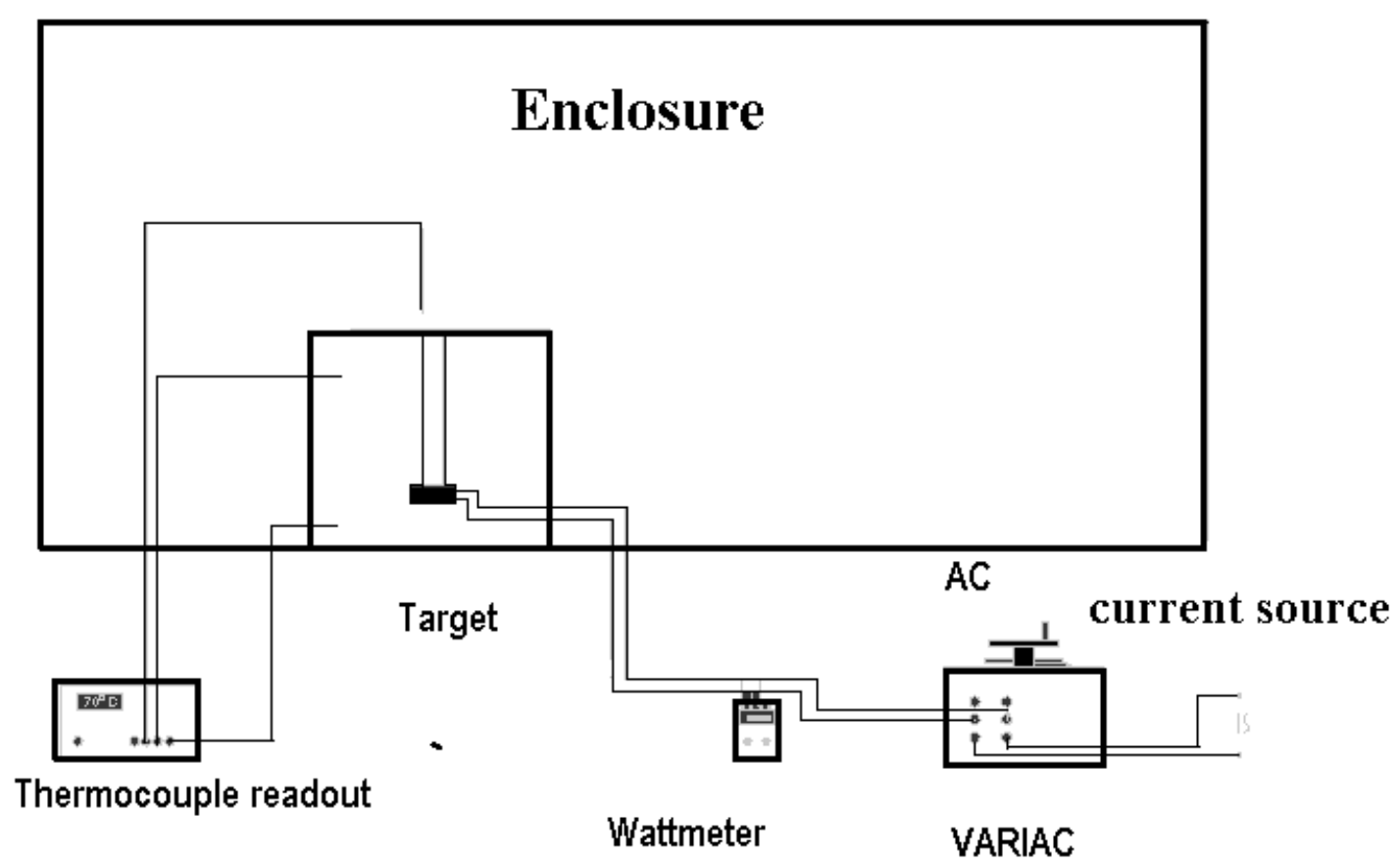

Figures (7) Schematic drawing for the test rig

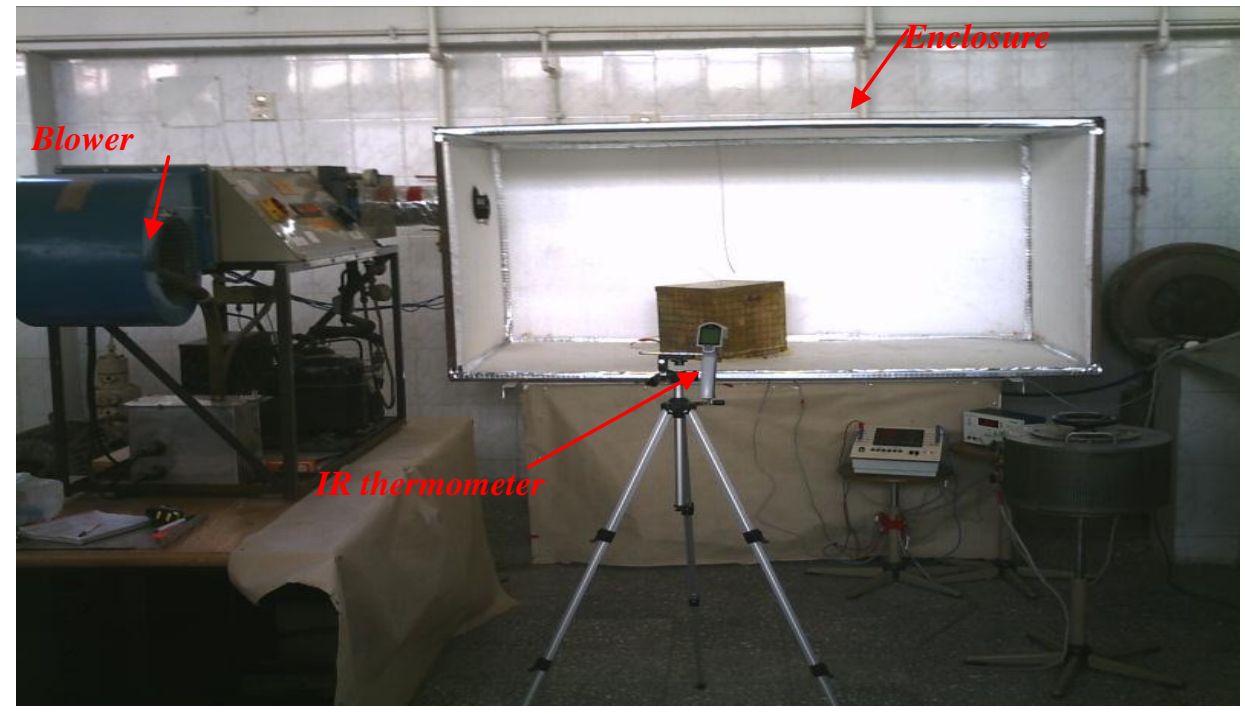

Figure (8) Photo of the test rig

Results of 1st computed model (figure (4)), and results from the captured image (figure (9)) have been analyzed by make comparisons on isothermal lines on the two results as shown in figure $(10-a, b, c)$. In line (1) there is a deviation in temperatures between the curve of 
computed model, and curve of captured image, although the two curves show the same trend. The cause of this deviation is the presence of the silicone material which covers the bottom edge of cube for the good insulation for heat. This new material of silicones has high emissivity than the emissivity of cube material which produces high temperatures. In line (2), the two curves show the same trends, except in the middle of the resulted curve of camera image there's a deviation due to presence of the fixing rivet of aluminum material. This rivet is of aluminum material which has high emissivity than the cube material that leads to a higher temperature. In line (3), the two curves show the same trends, although there's a decrease in temperature of the resulted curve of camera due to the overlapping of two edges at the top of the cube, which increase the thermal conductivity in the two overlapped edges, and this overlap didn't used in computation to simplify the problem.

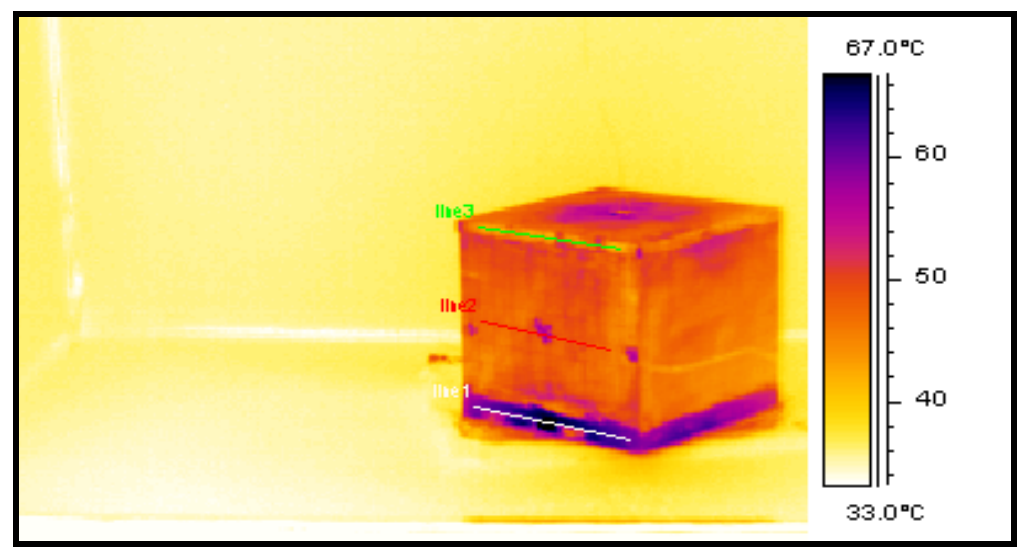

Figure (9): Image captured from the thermal camera for the 1st case study

Finally, these errors are presented exclusively for the previous isothermal lines. There are some general reasons that make mismatching in the whole figure for the two matched images in this study; these reasons are:

- Conditions of materials surface like surface roughness, thin or thick surface layers and oxide films on material surface have a severe impact on the radiation properties (real surface assumption)[5]. This fact leads to selecting of the assumption of grey diffuse surface in computation for simplifications. Thus, grey surface needs including of normal emissivity of material rather than including this property as a function of wavelength as well in the real surface.

- Making accurate models of targets geometry to match the real target is important, but this may causes some expenses in computational time and memory of computers.

- For long path of observation, including the atmospheric transmission is important, but for short path the atmospheric transmission is assumed to be 1 which simplifies the computations for producing zero distance quantities.

- Sensor sensitivity has an impact on the percentage error when validating the computational results with experimental results. This leads to importance of assisting sensor model and engage it with simulation models to get perfect predictions. The sensor accuracy of camera makes temperatures deviation of $\pm 20 \mathrm{C}[6]$. 


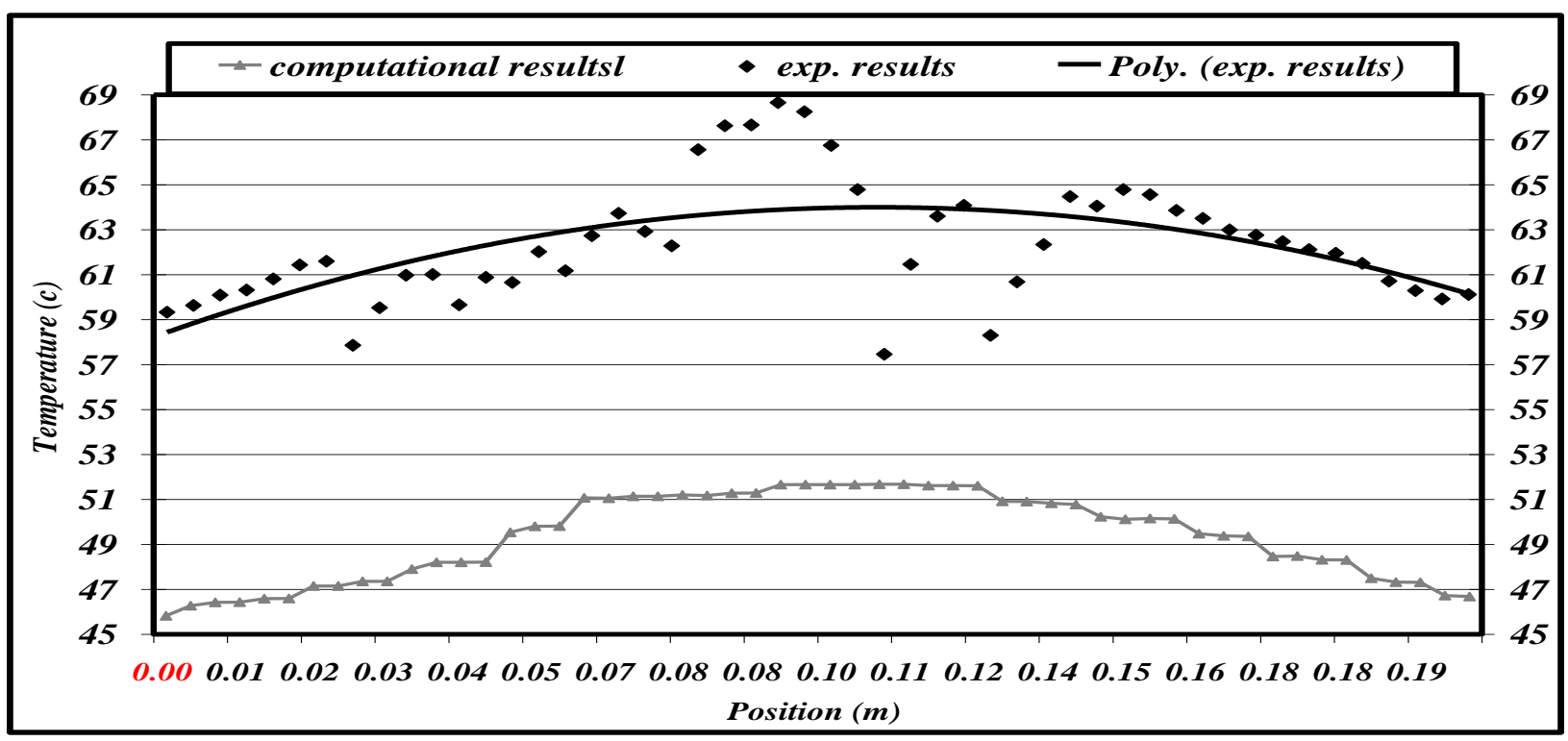

Figure (10a). Temperature trends on line 1 for 1 st case.

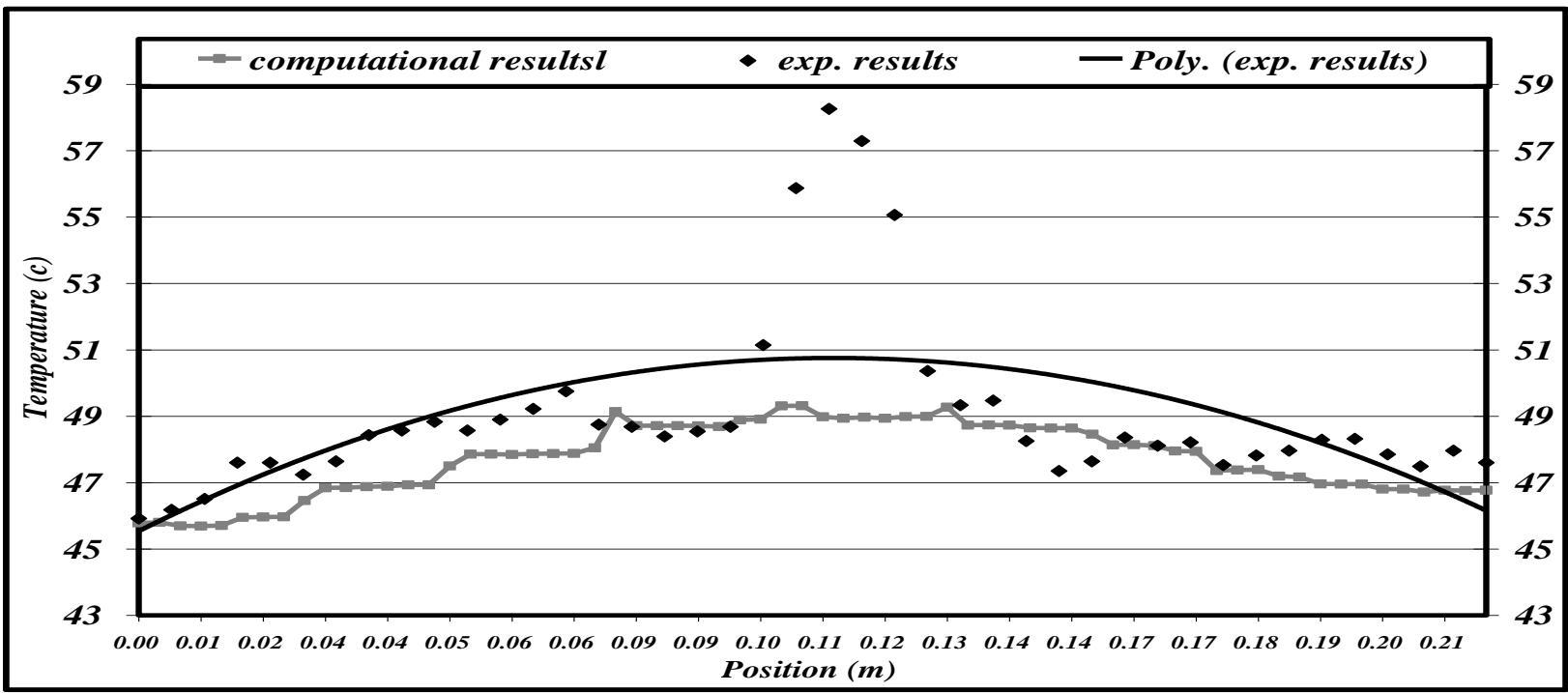

Figure (10b). Temperature trends on line 2 for 1 st case.

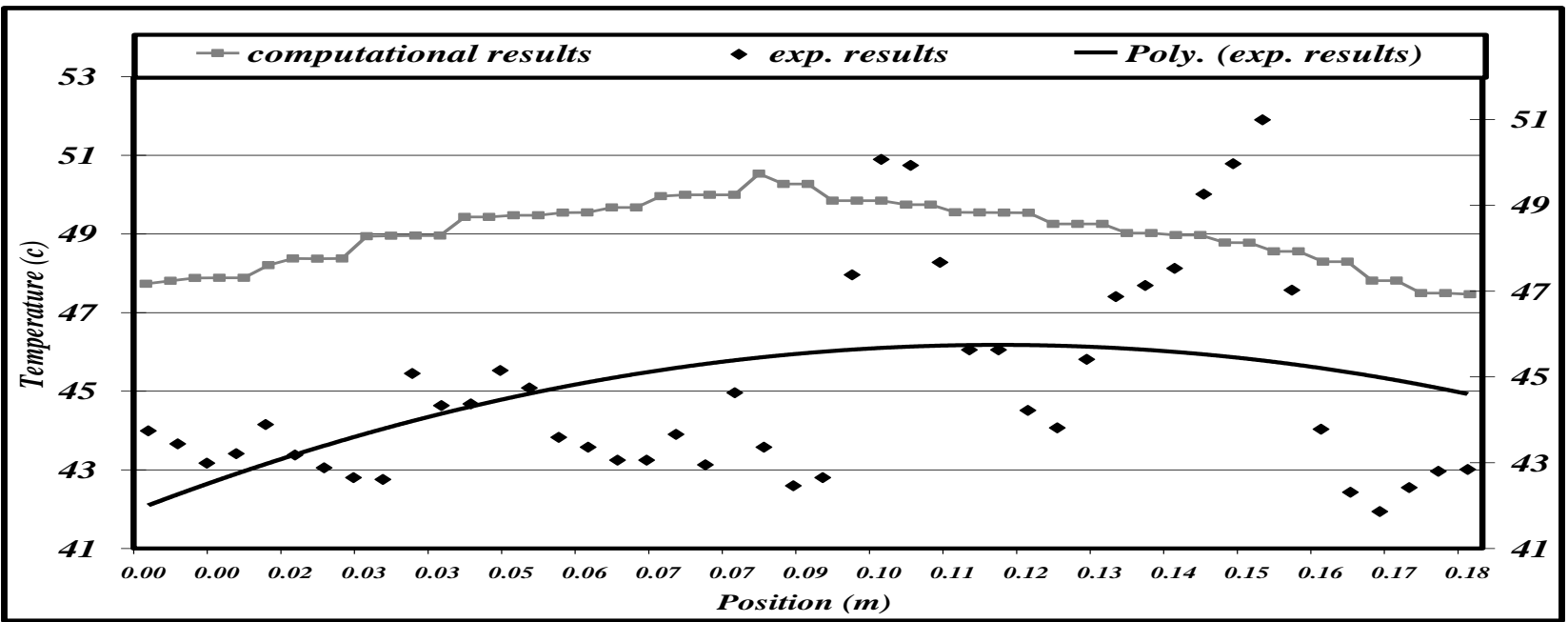

Figure (10c). Temperature trends on line 3 for 1 st case. 


\section{Conclusion}

This work introduces the importance of computer programs used as an alternative tool to facilitate getting the data required in characterizing and predicting thermal signature of targets. The modeling by computers requires knowing the constrained models form the prediction of thermal signature. These models are the target geometry model, heat transfer model, background model, atmospheric model and sensors model.

Thermal signature of targets means the combinations of target temperatures effects, background effect, atmospheric effects and sensor characteristics. This produces two terms of measured quantities; zero distance quantities (true signature due to target temperatures), and apparent quantities (contributions of the background and the atmosphere effects and also the sensor characteristic).

Geometry, thermal and flow conditions have a significant impact on studying heat transfer and geometry models. The study concludes different temperature distributions on objects surfaces due to the effects of objects, shapes, inside configurations of objects, the power and positions of internal heat sources and finally the external generated sources from the flow conditions.

The prediction of objects' thermal images by the specified package of the computational tool (FLUENT) shows satisfied compatibilities with experimental imaging prediction by infrared imaging tool. This confirms the abilities from using computers in the field of thermal signatures prediction and IR threats aspects in military fields. Although, presence of these compatibilities, some features of mismatching in both results have been appeared due to simplifying assumptions and measurements errors. The features of mismatching conclude some considerations should be taken into account.

In order to design a complete package of predicting the thermal signature of the targets, the work has shown the big need to design software can calculate the atmospheric transmission and path radiance at different field conditions. The engagements of all other considered models in predicting thermal signature (background model, atmospheric model, sensor model) is also important in designing a complete package for this purpose.

\section{References}

[1] HAMAMATSU PHOTONICS K.K., TECHNICAL INFORMATION SD-12, characteristics and use of infrared detectors, http://www.hamamatsu.com, Cat. No. KIRD9001E03, Nov. 2004 DN, Solid State Division.

[2] George J. Zissis, "Infrared \& Electro-Optical Systems Handbook", V.1, "Sources of Radiation", Ch.2, 1993, Infrared Information Analysis Center, Michigan and SPIE Optical Engineering Press, Washington.

[3] Fluent 6.3 User Guide". User Services Center for the latest release information.http://www.engres.odu.edu/Applications/fluent6.3/help/index.htm .

[4] J. P. Holman "Heat Transfer", Ninth Edition, October 2001, McGraw-Hill.

[5] Infrared training systems,(internet).www.infraredtraining.com.

[6] ThermaCAMTM Model (SC 2000), FLIR System AB, ThermaCAMTM Researcher User's Manual, , June, 2003, Sweden, http://www.flir.com. 